International Journal of Pure and Applied Mathematics

Volume 115 No. 4 2017, 827-834

ISSN: 1311-8080 (printed version); ISSN: 1314-3395 (on-line version)

url: http://www.ijpam.eu

doi: 10.12732 /ijpam.v115i4.16

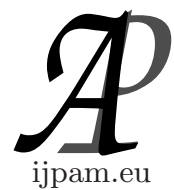

\title{
ON A CLASS OF PARA KENMOTSU MANIFOLDS
}

\author{
T. Satyanarayana ${ }^{1}$, K.L. Sai Prasad ${ }^{2}$, B. Satyanarayana ${ }^{3}$ \\ ${ }^{1}$ Department of Mathematics \\ Pragati Engineering College \\ Surampalem, Near Peddapuram, Andhra Pradesh, INDIA \\ ${ }^{2}$ Department of Mathematics \\ Gayatri Vidya Parishad College of Engineering for Women \\ Madhurawada, Visakhapatnam, 530 048, INDIA \\ ${ }^{3}$ Department of Mathematics \\ Acharya Nagarjuna University \\ Nagarjuna Nagar, Guntur, 522 510, INDIA
}

\begin{abstract}
The object of this paper is to investigate Weyl-pseudosymmetric para-Kenmotsu manifolds and para-Kenmotsu manifolds satisfying the condition $C(X, Y) . S=0$ where $C(X, Y)$ is the Weyl conformal curvature tensor and $S$ is the Ricci tensor of the manifold.
\end{abstract}

AMS Subject Classification: 53C15, 53C25

Key Words: Para Kenmotsu manifolds, pseudosymmetric manifold, conformal curvature tensor, Ricci tensor, scalar curvature.

\section{Introduction}

Sato [9] defined the notions of an almost para contact Riemannian manifold. After that, Adati and Matsumoto [1] defined and studied $p$-Sasakian and $s p$ Sasakian manifolds which are regarded as a special kind of an almost contact

Received: $\quad$ April 2, 2017

Revised: $\quad$ May 8, 2017

Published: August 8, 2017

$\S$ Correspondence author (c) 2017 Academic Publications, Ltd. url: www.acadpubl.eu 
Riemannian manifolds. Before Sato, Kenmotsu [8] defined a class of almost contact Riemannian manifolds. In 1995, Sinha and Sai Prasad [11] defined a class of almost para contact metric manifolds namely para Kenmotsu (briefly $p$-Kenmotsu) and special para Kenmotsu (briefly $s p$-Kenmotsu) manifolds. In a recent paper, the authors Satyanarayana and Sai Prasad [10] studied Weyl semisymmetric $p$-Kenmotsu manifolds, and they prove that such a manifold is conformally flat and hence is an $s p$-Kenmotsu manifold.

Let $\left(M_{n}, \mathrm{~g}\right)$ be an n-dimensional, $n \geq 3$, differentiable manifold of class $\mathrm{C}^{\infty}$. Let $\nabla$ be its Levi-Civita connection, $R$ be the Riemannian Christoffel curvature tensor and $C$ be the Weyl conformal curvature tensor and is defined by

$$
\begin{aligned}
C(X, Y) Z= & R(X, Y) Z \\
& -\frac{1}{n-2}[g(Y, Z) S X-g(X, Z) S Y+S(Y, Z) X-S(X, Z) Y] \\
& +\frac{r}{(n-1)(n-2)}[g(Y, Z) X-g(X, Z) Y],
\end{aligned}
$$

where $S$ is the Ricci tensor, $r$ is the scalar curvature of $M_{n}$ [3]. The Ricci operator $S$ and the (0,2)-tensor $S^{2}$ are defined by

$$
g(S X, Y)=S(X, Y)
$$

and

$$
S^{2}(X, Y)=S(S X, Y) .
$$

If $C=0$ and $n \geq 4$, then $M_{n}$ is conformally flat. If $\nabla \mathrm{C}=0$ then $M_{n}$ is called conformally symmetric, where $\nabla$ is Levi-Civita connection of the Riemannian metric [4]. It is obvious that a conformally symmetric manifold is Weyl-semisymmetric.

Furthermore we define the tensor $C(X, Y) . S$ on $M_{n}$ by

$$
(C(X, Y) . S)(Z, W)=-S(C(X, Y) Z, W)-S(Z, C(X, Y) W) .
$$

where $X, Y, Z, W \in \chi(M), \chi(M)$ being the Lie algebra of vector fields on $M_{n}$.

For a $(0, k)$-tensor field $\mathrm{T}, k \geq 1$, on $\left(M_{n}, \mathrm{~g}\right)$ we define the tensors $R$. $T$ and $Q(g, T)$ by

$$
\begin{array}{r}
(R(X, Y) . T)\left(X_{1}, X_{2}, \ldots, X_{k}\right)=-T\left(R(X, Y) X_{1}, X_{2}, \ldots, X_{k}\right) \\
-\ldots-T\left(X_{1}, \ldots, X_{k-1}, R(X, Y) X_{k}\right), \\
Q(g, T)\left(X_{1}, X_{2}, \ldots, X_{k} ; X, Y\right)=-T\left((X \wedge Y) X_{1}, X_{2}, \ldots, X_{k}\right) \\
-\ldots-T\left(X_{1}, \ldots, X_{k-1},(X \wedge Y) X_{k}\right)
\end{array}
$$


respectively [7], where the endomorphism $(X \wedge Y)$ is defined by

$$
(X \wedge Y) Z=g(Y, Z) X-g(X, Z) Y
$$

If the tensors $R$. $C$ and $Q(g, C)$ are linearly dependent then the manifold is called Weyl-pseudosymmetric [7]. This is equivalent to

$$
R . C=L_{C} Q(g, C)
$$

holding on the set $U_{C}=\{\mathrm{x} \in \mathrm{M} / \mathrm{C} \neq 0$ at $\mathrm{x}\}$, where $L_{C}$ is some function of $U_{C}$.

As a generalization of locally symmetric spaces, many geometers have considered semi-symmetric spaces and in turn their generalizations. Locally symmetric, semisymmetric and Pseudosymmetric Para-Sasakian manifolds are widely studied by many geometers $[2,5,6]$. In this study, our aim is to obtain the characterisations of the Weyl-pseudosymmetric para-Kenmotsu manifolds which are the extended classes of Weyl-semisymmetric para-Kenmotsu manifolds and para-Kenmotsu manifolds satisfying the condition $C(X, Y) . S=0$ where $C(X, Y)$ is the Weyl conformal curvature tensor and $S$ is the Ricci tensor of the manifold.

\section{2. $p$-Kenmotsu Manifolds}

Let $M_{n}$ be an $n$-dimensional differentiable manifold equipped with structure tensors $(\Phi, \xi, \eta)$ where $\Phi$ is a tensor of type $(1,1), \xi$ is a vector field, $\eta$ is a 1-form such that

$$
\begin{gathered}
\eta(\xi)=1 \\
\Phi^{2}(X)=X-\eta(X) \xi ; \bar{X}=\Phi X
\end{gathered}
$$

Then $M_{n}$ is called an almost para contact manifold.

Let $g$ be the Riemannian metric such that, for all vector fields $X$ and $Y$ on $M$

$$
\begin{gathered}
g(X, \xi)=\eta(X) \\
\Phi \xi=0, \eta(\Phi X)=0, \operatorname{rank} \Phi=n-1 \\
g(\Phi X, \Phi Y)=g(X, Y)-\eta(X) \eta(Y)
\end{gathered}
$$

Then the manifold $M_{n}$ [9] is said to admit an almost para contact Riemannian structure $(\Phi, \xi, \eta, g)$. 
A manifold of dimension $n$ with Riemannian metric $g$ admitting a tensor field $\Phi$ of type $(1,1)$, a vector field $\xi$ and a 1-form $\eta$ satisfying (2.1), (2.3) along with

$$
\begin{gathered}
\left(\nabla_{X} \eta\right) Y-\left(\nabla_{Y} \eta\right) X=0 \\
\left(\nabla_{X} \nabla_{Y} \eta\right) Z=[-g(X, Z)+\eta(X) \eta(Z)] \eta(Y)+[-g(X, Y)+\eta(X) \eta(Y)] \eta(Z) \\
\nabla_{X} \xi=\Phi^{2} X=X-\eta(X) \xi \\
\left(\nabla_{X} \Phi\right) Y=g(\Phi X, Y) \xi-\eta(Y) \Phi X
\end{gathered}
$$

is called a para Kenmotsu manifold or briefly $p$-Kenmotsu manifold [11].

A $p$-Kenmotsu manifold admitting a 1-form ' $\eta$ ' satisfying

$$
\left(\nabla_{X} \eta\right) Y=g(X, Y)-\eta(X) \eta(Y)
$$

$$
g(X, \xi)=\eta(X) \text { and }\left(\nabla_{X} \eta\right) Y=\varphi(\bar{X}, Y) \text {, where } \varphi \text { is an associate of } \Phi
$$

is called a special $p$-Kenmotsu manifold or briefly sp-Kenmotsu manifold [11].

It is known that [11] in a $p$-Kenmotsu manifold the following relations hold:

$$
\begin{gathered}
S(X, \xi)=-(n-1) \eta(X) \\
g[R(X, Y) Z, \xi]=\eta[R(X, Y, Z)]=g(X, Z) \eta(Y)-g(Y, Z) \eta(X) \\
R(\xi, X) Y=\eta(Y) X-g(X, Y) \xi \\
R(X, Y, \xi)=\eta(X) Y-\eta(Y) X ; \text { when } X \text { is orthogonal to } \xi
\end{gathered}
$$

where $S$ is the Ricci tensor and $R$ is the Riemannian curvature.

If the Ricci curvature tensor $S$ is of the form

$$
S=a I_{d}+b \eta \otimes \xi
$$

where $a$ and $b$ are smooth functions on $M_{n}$, then $M_{n}$ is called as an $\eta$-Einstein manifold and if $b=0$ then it is an Einestein manifold [2].

The above results will be used further in the next section. 


\section{Main Results}

In this section our aim is to find the characterisation of para-Kenmotsu manifolds satisfying the condition $C(X, Y) . S=0$ and Weyl-pseudosymmetric paraKenmotsu manifolds.

Firstly we give the following proposition.

Proposition 3.1. Let $M_{n}(n \geq 4)$ be an $n$-dimensional $p$-Kenmotsu manifold. If the condition $C(X, Y) . S=0$ holds on $M_{n}$ then

$$
S^{2}(X, Y)=\left[\frac{r}{(n-1)}-n+2\right] S(X, Y)+[r+n-1] g(X, Y) \text {. }
$$

Proof. Assume that $M_{n}$ is an $n$-dimensional, $n \geq 4, p$-Kenmotsu manifold satisfying the condition $C(X, Y) . S=0$.

Then from (1.4) we have

$$
S(C(U, X) Y, Z)+S(Y, C(U, X) Z)=0 .
$$

Putting $U=\xi$ in $(3.2)$ we have

$$
S(C(\xi, X) Y, Z)+S(Y, C(\xi, X) Z)=0 .
$$

By using (1.1), (2.12) and (2.13), (3.3) reduces to

$$
\begin{aligned}
& \eta(Y) S(X, Z)-g(X, Y) S(\xi, Z)+\eta(Z) S(X, Y)-g(X, Z) S(\xi, Y) \\
& \quad-\frac{1}{(n-2)}\left[S(X, Y) S(\xi, Z)-S(X, Z) S(\xi, Y)+g(X, Y) S^{2}(\xi, Z)\right. \\
& -\eta(Y) S^{2}(X, Z)+S(X, Z) S(\xi, Y)-S(X, Y) S(\xi, Z)+g(X, Z) S^{2}(\xi, Y) \\
& \left.\quad-\eta(Z) S^{2}(X, Y)\right]+\frac{r}{(n-1)(n-2)}[g(X, Y) S(\xi, Z)-\eta(Y) S(X, Z) \\
& \quad+g(X, Z) S(\xi, Y)-\eta(Z) S(X, Y)]=0 .
\end{aligned}
$$

Hence by the use of (1.3) and (2.12) we get

$$
\begin{aligned}
& \eta(Y) S(X, Z)-(1-n) g(X, Y) \eta(Z)+\eta(Z) S(X, Y)-(1-n) g(X, Z) \eta(Y) \\
& \quad-\frac{1}{(n-2)}\left[-\eta(Y) S^{2}(X, Z)-\eta(Z) S^{2}(X, Y)+(1-n)^{2} \eta(Z) g(X, Y)\right. \\
& \left.\quad+(1-n)^{2} \eta(Y) g(X, Z)\right]+\frac{r}{(n-1)(n-2)}[-\eta(Y) S(X, Z)-\eta(Z) S(X, Y)] \\
& \quad+(1-n)[\eta(Z) g(X, Y)+\eta(Y) g(X, Z)]=0 .
\end{aligned}
$$


Now by putting $Z=\xi$ in (3.5) and on using (1.3) and (2.12), we obtain

$$
\begin{aligned}
& \frac{1}{(n-2)} S^{2}(X, Y)=\left[\frac{r}{(n-1)(n-2)}-1\right] S(X, Y) \\
& +\left[\frac{r}{(n-2)}+\frac{(n-1)^{2}}{(n-2)}-(n-1)\right] g(X, Y),
\end{aligned}
$$

which is the required eqn (3.1), since $n \geq 4$.

Now, let us consider an $\eta$-Einstein $p$-Kenmotsu manifold. Then we can write the Ricci tensor $S$ as

$$
S(X, Y)=a g(X, Y)+b \eta(X) \eta(Y)
$$

where $X$ and $Y$ are any vector fields and $a$ and $b$ are smooth functions on $M_{n}$.

On contracting (3.7), we have

$$
r=n a+b,
$$

where $r$ is the scalar curvature.

On the other hand, putting $X=Y=\xi$ in (3.7) and on using (2.12), we also have

$$
1-n=a+b
$$

Hence it follows from (3.7), (3.8) and (3.9) that the Ricci tensor $S$ of an $\eta$ Einstein $p$-Kenmotsu manifold is given by

$$
S(Y, Z)=\left(1+\frac{r}{n-1}\right) g(Y, Z)+\left(\frac{r}{1-n}-n\right) \eta(Y) \eta(Z)
$$

Proposition 3.2. Let $M_{n}(n \geq 4)$ be an $n$-dimensional $\eta$-Einstein $p$ Kenmotsu manifold. Then the condition $C(X, Y) . S=0$ holds on $M_{n}$.

Proof. Assume that $M_{n}$ is an $\eta$-Einstein $p$-Kenmotsu manifold. Since the Weyl tensor $C$ has all symmetries of a curvature tensor, then from (1.4) it is easy to show that

$$
(C(U, X) . S)(Y, Z)=\left(\frac{r}{1-n}-n\right)[\eta(C(U, X) Y) \eta(Z)+\eta(C(U, X) Z) \eta(Y)]
$$

for all vector fields $U, X, Y, Z$ on $M_{n}$.

On using $(1.1),(2.12),(2.13)$ and $(3.10),(3.11)$ reduces to $(C(U, X) . S)(Y, Z)=$ 0 , which proves the proposition. 
Theorem 3.1. Let $M_{n}$ be an $n$-dimensional ( $\left.n \geq 4\right)$-Kenmotsu manifold. If $M_{n}$ is Weyl-pesudosymmetric then $M_{n}$ is either conformally flat, in which case $M_{n}$ is a SP-Kenmotsu manifold, or $L_{C}=-1$ holds on $M_{n}$.

Proof. Assume that $M_{n}$ is an $n$-dimensional $(n \geq 4)$ Weyl-pseudosymmetric $p$-Kenmotsu manifold and $X, Y, U, V, W \in \chi\left(M_{n}\right)$. Then from (1.8) we have

$$
(R(X, Y) . C)(U, V, W)=L_{C} Q(g, C)(U, V, W ; X, Y) .
$$

On using (1.5) and (1.6), we write (3.12) as:

$$
\begin{gathered}
R(X, Y) C(U, V) W-C(R(X, Y) U, V) W-C(U, R(X, Y) V) W \\
-C(U, V) R(X, Y) W=L_{C}[(X \wedge Y) C(U, V) W-C((X \wedge Y) U, V) W \\
-C(U,(X \wedge Y) V) W-C(U, V)(X \wedge Y) W] .
\end{gathered}
$$

Now by replacing $X$ with $\xi$ in $(3.13)$ we have

$$
\begin{aligned}
R(\xi, Y) C(U, V) W-C & (R(\xi, Y) U, V) W-C(U, R(\xi, Y) V) W \\
-C(U, V) R(\xi, Y) W & =L_{C}[(\xi \wedge Y) C(U, V) W-C((\xi \wedge Y) U, V) W \\
& -C(U,(\xi \wedge Y) V) W-C(U, V)(\xi \wedge Y) W] .
\end{aligned}
$$

So on using $(2.13),(1.7)$ and by taking the inner product of (3.14) with $\xi$, we get

$$
\begin{array}{r}
{\left[1+L_{C}\right][-\eta(Y) \eta(C(U, V) W)+C(U, V, W, Y)+\eta(U) \eta(C(Y, V) W)} \\
-g(Y, U) \eta(C(\xi, V) W)+\eta(V) \eta(C(U, Y) W)-g(Y, V) \eta(C(U, \xi) W) \\
+\eta(W) \eta(C(U, V) Y)-g(Y, W) \eta(C(U, V) \xi]=0 .
\end{array}
$$

Putting $Y=U$ in (3.15) and on contraction with respect to $U,(3.15)$ gives us

$$
\left(1+L_{C}\right)[\eta(C(\xi, V) W)]=0 .
$$

If $L_{C}=0$ then $M_{n}$ is Weyl-semisymmetric and so the equation reduces to

$$
\eta(C(\xi, V) W)=0
$$

which gives (3.10), shows that $M_{n}$ is an $\eta$-Einstein manifold.

Now on using (3.17) and (3.10), (3.15) takes the form $C(U, V, W, Y)=0$, means that the para-Kenmotsu manifold $M_{n}$ is conformally flat and hence by [10], $M_{n}$ is a SP-Kenmotsu manifold.

If $L_{C} \neq 0$ and $\eta(C(\xi, V) W) \neq 0$ then $\left(1+L_{C}\right)=0$, which gives $L_{C}=-1$. This completes the proof of the theorem. 
So we have the following corollary.

Corollary 3.1. Every $n$-dimensional, $n \geq 4$, para-Kenmotsu manifold is a Weyl-pesudosymmetric manifold of the form $R$. C $=-Q(g, C)$.

Remark. The above findings are quite in similar to the results obtained for Weyl-pseudosymmetric para-Sasakian manifolds [5].

\section{Acknowledgments}

The authors acknowledge Prof. Kalpana of Banaras Hindu University, Varanasi and Dr. A. Kameswara Rao, Assistant Professor of G.V.P. College of Engineering for Women, Visakhapatnam for their valuable suggestions in preparation of the manuscript.

The authors declare that there is no conflict of interests regarding the publication of this paper.

\section{References}

[1] T. Adati and K. Matsumoto, On conformally recurrent and conformally symmetric PSasakian manifolds, TRU Math., 13 (1977), 25-32.

[2] T. Adati and T. Miyazawa, On P-Sasakian manifolds satisfying certain conditions, Tensor (N.S.), 33, (1979), 173-178.

[3] R. L. Bishop and S. I. Goldberg, On conformally flat spaces with commuting curvature and Ricci transformations, Canad. J. Math., 14, No.5 (1972), 799-804, doi: 10.4153/CJM-1972-077-6.

[4] M. C. Chaki and B. Gupta, On conformally symmetric spaces, Indian J. Math., 5, (1963), 113-122.

[5] Cihan Ozgur, On a class of Para-Sasakian Manifolds, Turk J. Math., 29, (2005), 249-257.

[6] U. C. De and D. Tarafdar, On a type of P-Sasakian manifold, Math. Balkanica (N.S.), 7, (1993), 211-215.

[7] R. Deszcz, On Pseudosymmetric spaces, Bull. Soc. Math. Belg., 49, (1990), 134-145.

[8] K. Kenmotsu, A class of almost contact Riemannian manifolds, Tohoku Math. Journal, 24 (1972), 93-103, doi: 10.2748/tmj/1178241594.

[9] I. Sato, On a structure similar to the almost contact structure, Tensor (N.S.), 30 (1976), 219-224.

[10] T. Satyanarayana and K.L. Sai Prasad, On a type of Para Kenmotsu Manifold, Pure Mathematical Sciences, 2, No.4 (2013), 165-170.

[11] B. B. Sinha and K. L. Sai Prasad, A class of almost para contact metric Manifold, Bull. of the Calcutta Math. Soc., 87 (1995), 307-312. 